\title{
Research
}

\section{Urgent suspected cancer referrals from general practice:}

\author{
audit of compliance with guidelines and referral outcomes
}

\begin{abstract}
\section{Background}

Late diagnosis contributes to the UK having poorer cancer survival than many countries in Europe. Cancer referral guidelines help GPS decide which patients to refer urgently for further investigation
\end{abstract}

\section{Aim}

To examine primary care referral patterns, compliance with referral guidance, and eventual outcome for patients

\section{Design and setting}

Prospective audit within general practice in Scotland.

\section{Method}

GPs in Scotland reviewed all urgent suspected cancer referrals over a 6 -month period. They noted the final diagnosis and assessed whether the referral was in accordance with agreed referral guidelines

\section{Results}

A total of 18775 urgent suspected cancer referrals were analysed from 516 GP practices. The referral rate ranged from 3.7 to 24.0 per 1000 per annum; $30.8 \%$ of referrals were for patients aged under 50 years, yet this age group accounts for only $11.1 \%$ of all diagnosed cancers; $10.3 \%$ of all urgent cancer referrals were for suspected melanoma, despite this cancer accounting for only $4.1 \%$ of new cancers. The proportion of patients subsequently diagnosed with cancer was greatest for leukaemia (61.7\%), prostate (52.6\%), and lung cancer referrals (39.7\%), and lowest for melanoma (11.8\%), oesophago-gastric (11.2\%), brain (10.6\%), and laryngeal cancer referrals (7.8\%). Compliance with referral guidelines was $90.9 \%$. A large proportion of referrals considered to be outside the guidelines still had a cancer diagnosed lurological 15.9\%, lung 8.8\%, colorectal $8.4 \%$, and breast $6.4 \%$ ).

\section{Conclusion}

There is wide variation in GP referral rates for suspected cancer with a greater than expected proportion of referrals for younger people. Many referrals considered to be outside the national guidelines were diagnosed with cancer, suggesting factors other than those in referral guidelines alert GPs to the possibility of cancer.

\section{Keywords}

guidelines as topic; neoplasm; primary care, referral and consultation.

\section{INTRODUCTION}

In the UK, there is relatively poor survival from cancer in comparison with some other European countries. ${ }^{1}$ Cancer experts believe that this is due to a more advanced stage at diagnosis, with delays between the onset of symptoms, presentation to primary care, and referral onwards playing contributory roles. ${ }^{2}$ GPs have an important role in referring patients with symptoms or signs of cancer for further investigation. This can be a difficult task because most cancer symptoms are not specific to cancer. Delays in diagnosis may also occur when GPs mistakenly attribute symptoms, signs, or abnormal test results to a health problem other than cancer. ${ }^{3}$

To aid appropriate referral, nationally agreed guidelines have been published to help GPs decide which patients should be referred urgently for further investigation. ${ }^{4-}$ ${ }^{6}$ These referral guidelines are based on a mixture of evidence and consensus among experts.

This paper reports on a prospective audit of urgent suspected cancer referrals by GPs, examining referral patterns from individual GP practices, compliance with referral guidelines, and eventual outcome. This follows previous work undertaken within primary care in Scotland where GPs had been asked to audit the referral pathway for all new cancer diagnoses, examining whether the referral had been made urgently lwhere cancer was suspected), or routinely (where cancer may not have been suspected).?

P Baughan, MRCGP, Lead Cancer GP, Falkirk Community Hospital, Westburn Avenue, Falkirk, Scotland. J Keatings, BSc, information officer, West of Scotland Cancer Network, Glasgow Royal Infirmary, Glasgow. B O'Neill, FRCGP, lead cancer GP, Astley Ainsley Hospital, Edinburgh.

\section{Address for correspondence}

Paul Baughan, NHS Forth Valley, Lead Cancer Team, Administration Block, Falkirk Community

\section{METHOD}

In early 2008, GPs in 9 of the 11 mainland health boards in Scotland agreed to review all urgent suspected cancer referrals, excluding non-melanotic skin cancers, within their practice during a defined 6 month period. Engagement with the study was facilitated by the enhanced service component of the General Medical Services contract, which enabled a payment to be made for participating practices.

An Excel spreadsheet template with defined parameters and preset drop-down options was provided to participating practices, with instructions on its use. The template was locked to prevent changes being made to it. The parameters requested were: age at referral, sex, and specific cancer suspected (bladder, brain, breast, cervical, colorectal, endometrial, gallbladder, laryngeal, leukaemia, liver, lung, lymphoma, melanoma, mesothelioma, myeloma, oesophageal, oropharyngeal, ovarian, pancreatic, prostate, renal, sarcoma, small intestine, stomach, testicular, thyroid, vulval, other, no information, and not certain). Other parameters required were eventual diagnosis made (the suspected cancer, another cancer, or no cancer), compliance with guidelines, route of referral, use of a referral proforma, and any perceived delays in communication from secondary care. Space was left for additional comments.

Data from the practice spreadsheets were collated into a single Microsoft Access database, and tumours were grouped

Hospital, Westburn Avenue, Falkirk, FK1 5SU.

E-mail: paul.baughananhs.net

Submitted: 8 November 2010; Editor's response: 12 January 2011; final acceptance: 6 April 2011. CBritish Journal of General Practice

This is the full-length article (published online 31 Oct 2011) of an abridged version published in print. Cite this article as: Br J Gen Pract 2011; DOI: 10.3399/bjgp11X606591. 


\section{How this fits in}

GPs within the UK are encouraged to use cancer-referral guidelines when deciding who to refer urgently with suspected cancer. The evidence base behind many of the cancer-referral guidelines is poor. This audit demonstrates a wide variation in both the pattern of referral and also the compliance with referral guidelines, depending on factors such as individual GP practice, patient age, and suspected tumour type. These results support the need to improve the evidence base and refine the referral guidelines for suspected cancer.

together as appropriate (for example, liver pancreatic, and gallbladder grouped as hepatobiliary. Once collated, a number of quality checks were carried out on the data, and some of the data provided were excluded from the analyses. Excluded cases comprised: cases where the information on cancer suspected was not completed, cases where the cancer suspected was a non-melanotic skin cancer, and cases from practices that reported that $100 \%$ of their urgent suspected cancer referrals had an eventual diagnosis of cancer las it was assumed that these practices had misinterpreted the initial instructions).

Practice list sizes were used to calculate the number of referrals per 1000 population. These list sizes were supplied by each practice at the time of data collection. Analysis of data was carried out using Microsoft Access and SPSS (version 17.0).

\section{RESULTS}

A total of 562 GP practices from 9 of the 11 Scottish mainland health boards (covering approximately $59.3 \%$ of the Scottish population) submitted data on 19574 urgent referrals. Of the total number of referral records submitted, $4.1 \%$ had to be excluded from analysis, $1.9 \%$ of which were cases where there was no information entered regarding the suspected cancer diagnosis. After exclusions, 18775 patients from 516 practices were included in the analysis.

When the practices with the highest and lowest $10 \%$ of referral rates were excluded, there remained a six-fold variation in referral rate, from 3.7 urgent referrals per 1000 population per year to 24.0 urgent referrals per 1000 population per year (Figure 1).

When the 18775 patients were split into the suspected diagnosis at the time of urgent referral, suspected breast cancer I $=3436,18.3 \%)$ and colorectal cancer $(n=$ $3370,17.9 \%$ ) were the two most likely reasons, by far, for a GP to refer a patient, accounting for $36.3 \%$ of all referrals.

There was no apparent association between the proportion of urgent referrals and cancer incidence for the five most common cancers (Figure 2). ${ }^{8}$ Lung cancer accounted for $16.8 \%$ of newly diagnosed cancers in Scotland in 2008, yet only 5.6\% In $=1045$ ) of urgent referrals; whereas melanoma accounted for $4.1 \%$ of newly diagnosed cancers and $10.3 \%(n=1942)$ of all urgent referrals. ${ }^{8}$

The proportion of all urgent cancer referrals with an eventual diagnosis of cancer leither the cancer suspected or a different cancer) was $18.3 \%(n=3432)$. This varied according to tumour group. Over $50 \%$

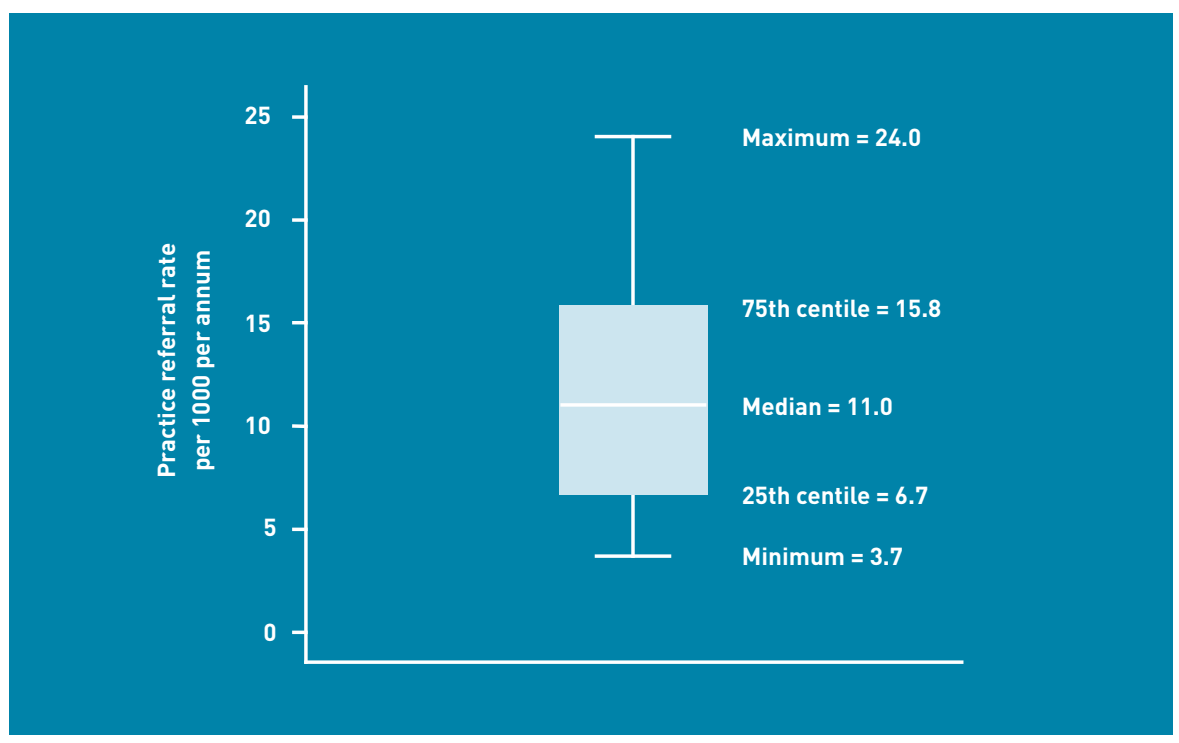




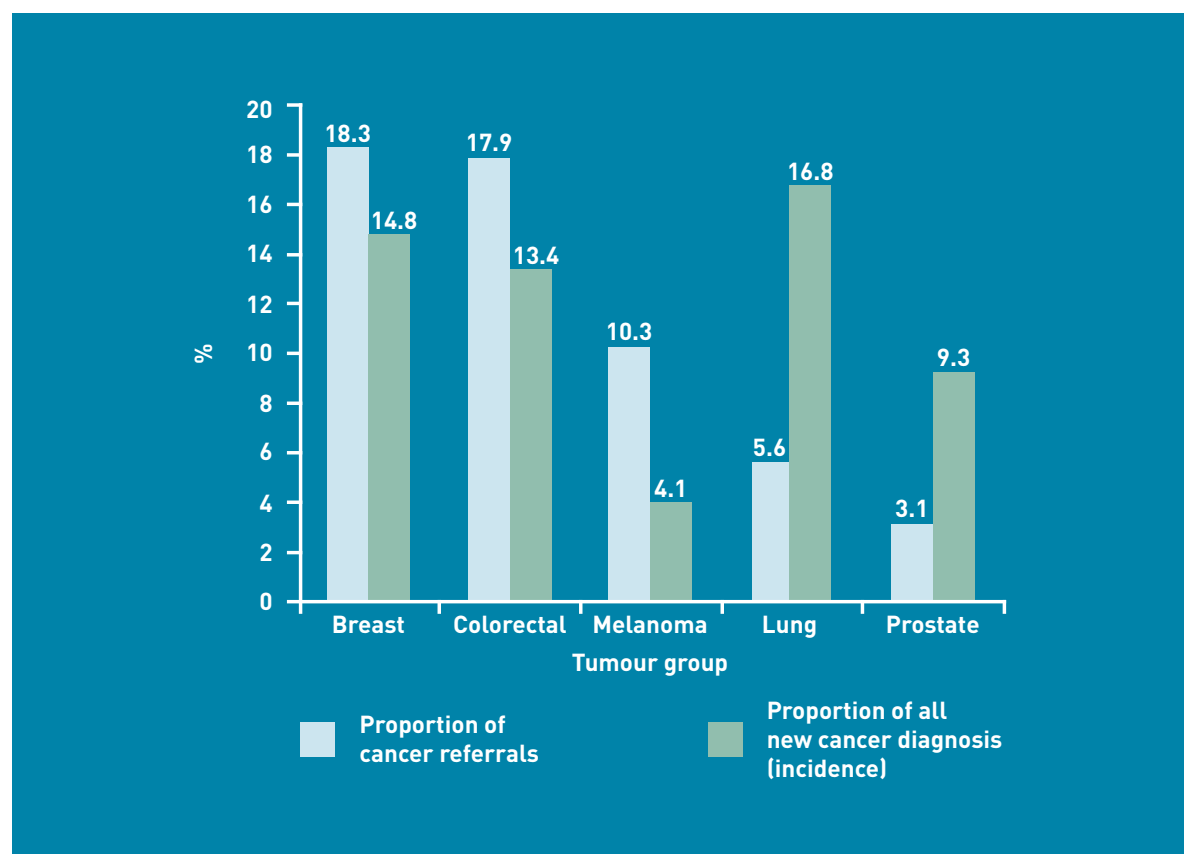

Figure 2. Comparison between cancer referrals and incidence by tumour group.

Figure 3. Proportion of referrals with an eventual diagnosis of cancer by tumour group.

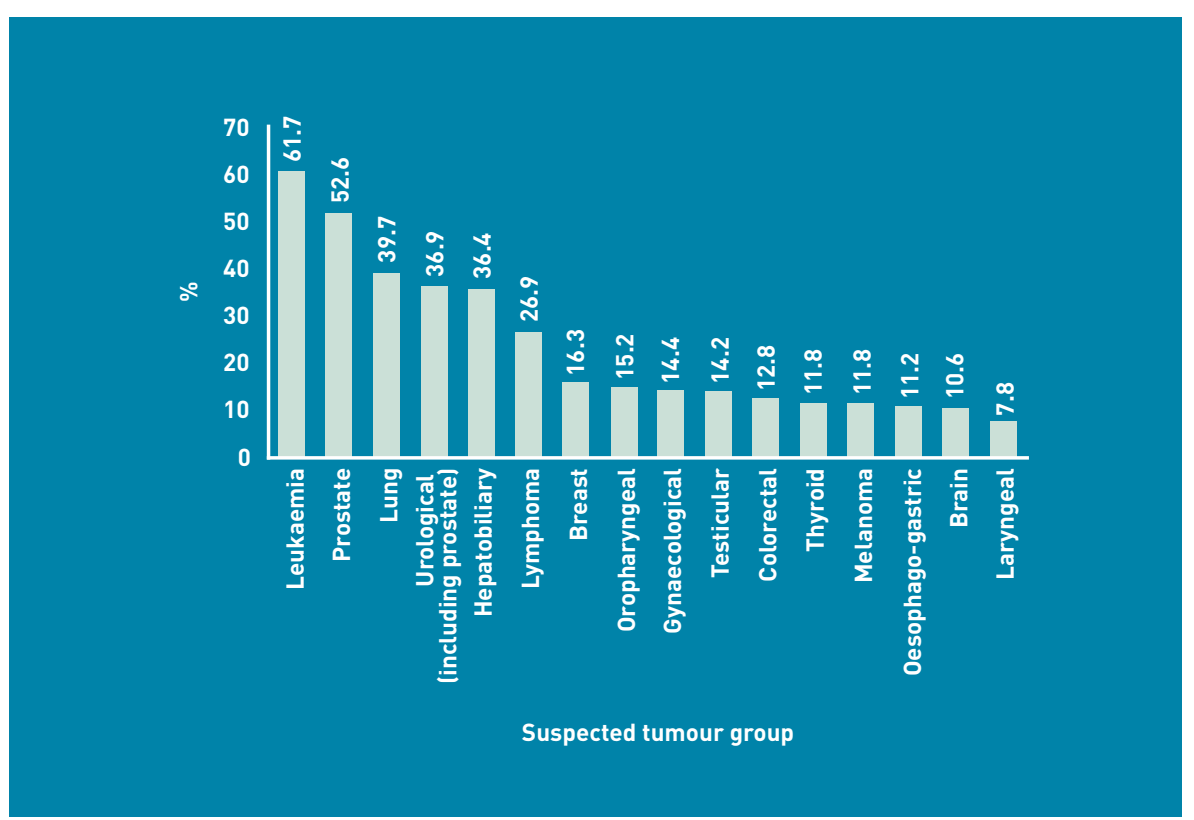

the younger age groups (Figure 4). Of all urgent cancer referrals, 30.8\% (5766/18 710) were for patients aged under 50 years, compared with only $11.1 \%$ of newly diagnosed cancers coming from this age group. Conversely, only 10.8\% (2015/18 710) of cancer referrals were for patients aged over 80 years, while this age group accounted for $19.7 \%$ of all cancers diagnosed. 8

The age of the patient at the time of urgent suspected cancer referral had an important bearing on whether a cancer was diagnosed (Figure 5), with 2.4\% (7/296) of referrals of all patients under the age of 20 years having a diagnosis of cancer compared with $30.3 \%$ (611/2015) of referrals of all patients aged 80 years and over.

GPs were asked to review their referrals with reference to the national cancerreferral guidelines, and assess whether they considered the urgent referral to be in accordance with these guidelines. The overall compliance rate was $90.9 \%$ (14937/16441), ranging from 94.0\% (529/563) for patients with suspected laryngeal cancer to $75.0 \%$ (45/60) for those with suspected leukaemia. Compliance with guidelines was greater than $85 \%$ for every tumour group except testicular cancer (84.9\%; 197/232), brain cancer (78.3\%; 126/161), hepatobiliary (77.3\%; 119/154), and leukaemia (75.0\%; 45/60).

When GPs reviewed the eventual outcome following a suspected cancer referral, there was a greater proportion of patients diagnosed with cancer $18.1 \%$; 2700/14937) if the GP referred in accordance with the suspected cancerreferral guidelines; however, 7.7\% (56/724) of all suspected cancer referrals that were considered by the GP to be outside the national referral guidelines had an eventual diagnosis of cancer (Figure 6).

\section{DISCUSSION}

\section{Summary}

This study has yielded valuable information about the referral patterns and eventua outcome for 18775 patients referred with suspected cancer in Scotland during a 6month period in early 2008

The audit reveals a wide variation in the referral rate between different GP practices across Scotland. Patient demographics vary between GP practices; however, even if this is taken into account by excluding the GP practices with the highest and lowest $10 \%$ of referral rates, there was still a six-fold difference in the total number of urgent suspected cancer referrals per 1000 population between the GP practices. 
Figure 4. Proportion of referrals compared with published data on cancer incidence by age.
Figure 5. Proportion of cancer referrals with an eventual diagnosis of cancer, by patient age.

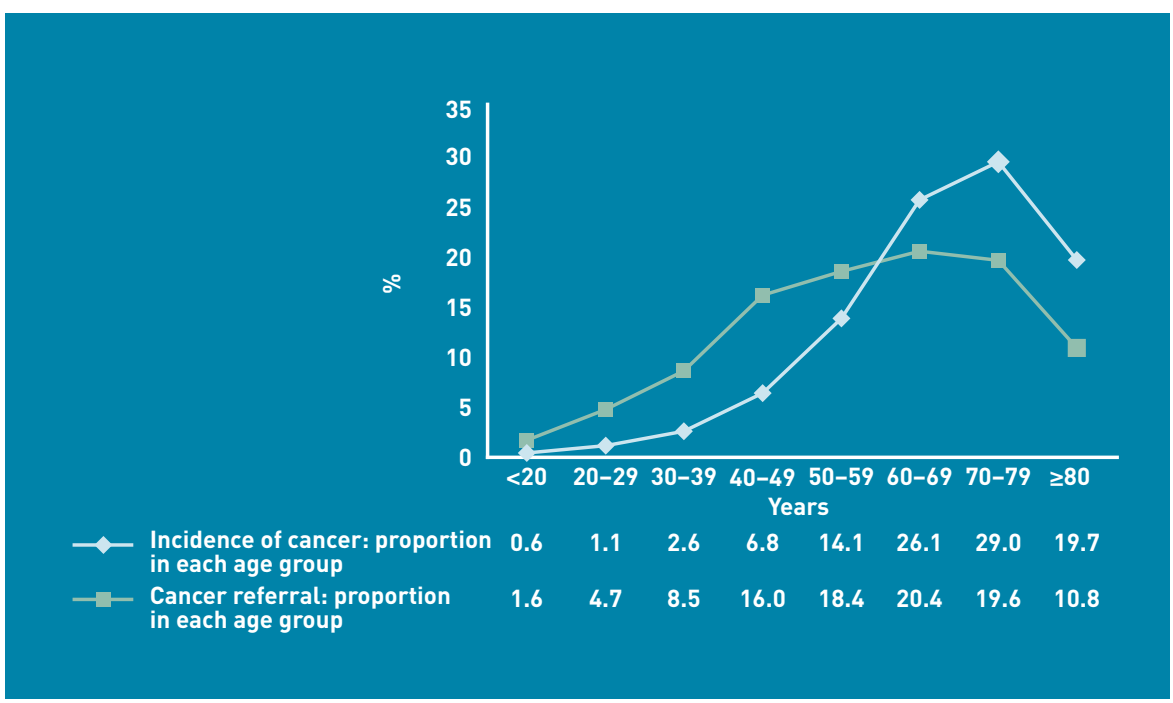

Detecting cancer early can be notoriously difficult, due to the subtle and non-specific nature of many symptoms. This variation in referral rate suggests that there are significant differences in the thresholds for individual GPs to refer symptomatic patients for further investigation.

From an economic perspective, it is tempting to criticise the highest-referring GP practices for over-investigating patients and using valuable resources that might be better used elsewhere. However, it may be equally important to focus attention on the lowest-referring practices, as there is a risk that they are missing some cancers. With the use of electronic referral systems, now widespread in Scotland, it is much easier to obtain comparative referral data at individual practice level. It is important that practices have easy access to these data and that they are encouraged to review their referral patterns and, if applicable, examine reasons for lying at either end of the referral curve.

The proportion of referrals for lung cancer $(5.6 \%)$ and prostate cancer $(3.1 \%)$ was much lower than might be expected. Patients with symptoms of lung cancer will often have had a chest X-ray and computerised tomography (CT) prior to referral. Similarly, patients with prostatic symptoms will usually have had a prostatespecific antigen (PSA) blood test prior to any decision to refer. Access to these diagnostic tests allows GPs to reassure some symptomatic patients that referral is not necessary as cancer is unlikely.

The 'pick up' rate or proportion of referrals with an eventual diagnosis of cancer (Figure 3), was greatest for suspected leukaemia (61.7\%), and prostate $(52.6 \%)$ and lung cancer $(39.7 \%)$, and lowest for melanoma (11.8\%), and oesophagogastric $(11.2 \%)$, brain (10.6\%), and laryngeal cancers $(7.8 \%)$. This may also reflect the availability of and direct access to diagnostic investigations (for example, full blood count, PSA, ultrasound, chest X-ray, CT chest), allowing the referring GP to get additional information regarding the likelihood of cancer prior to referral. Changes in direct access to other diagnostic investigations (for example, upper and lower gastrointestinal endoscopy and wider access to CT or magnetic resonance imaging [MRI]] may, in the future, help GPs to identify an increasing number of patients with cancer.

When the urgent referrals were split into tumour groups based on the suspected

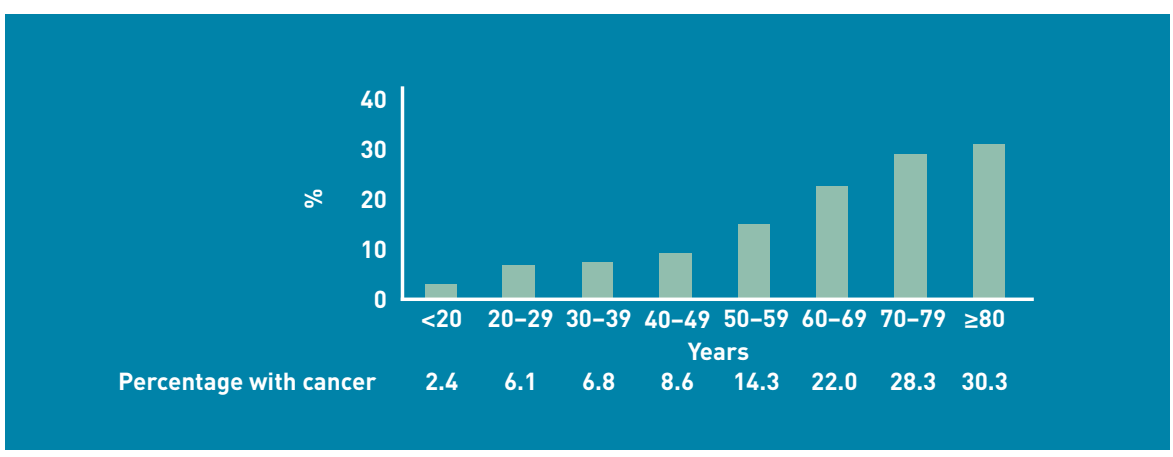




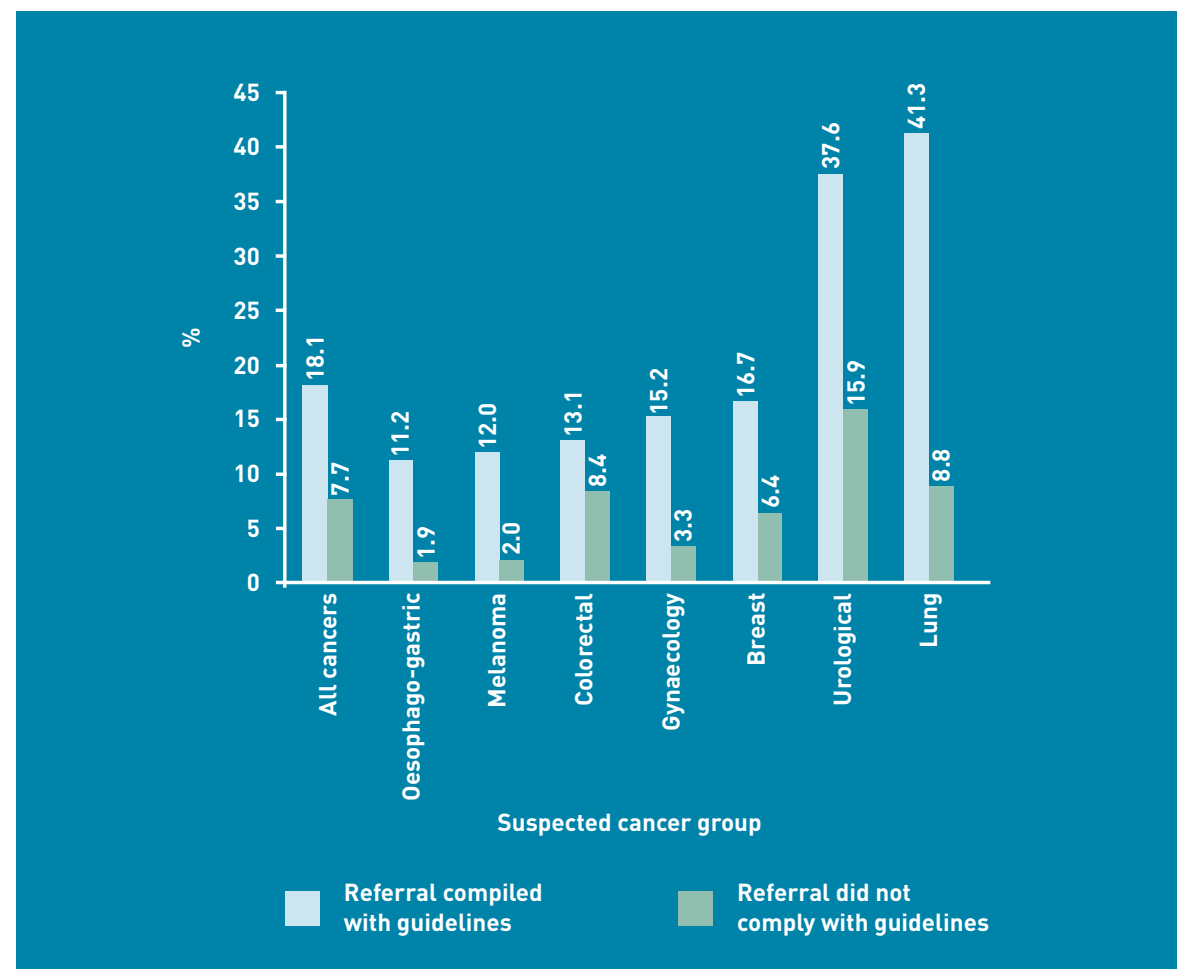

Figure 6. Proportion of referrals with an eventual diagnosis of cancer by compliance with guidelines.

\section{Funding}

Practices were funded to complete the audit using the enhanced services component of the UK General Medical Services contract.

\section{Provenance}

Freely submitted; externally peer reviewed.

\section{Ethical approval}

Ethical approval was not required as no identifiable patient information was provided.

\section{Competing interests}

The authors have declared no competing interests.

\section{Acknowledgements}

We are grateful to GP colleagues who conducted the audit, and specifically to members of the Scottish Primary Care Cancer Group who supported the process. Thanks to Rachel Whitton, NHS Forth Valley, for designing the reporting template, and to Eilidh Fletcher, Information Officer, Health Intelligence Unit, NHS Lothian, for help in the collation and analysis of the data.

\section{Discuss this article}

Contribute and read comments about this article on the Discussion Forum: http://www.rcgp.org.uk/bjgp-discuss cancer, melanomas accounted for a much higher proportion of referrals than might have been expected. Malignant melanoma was the third most common reason for referral (with $10.3 \%$ of all referrals) although it accounted for only $4.1 \%$ of all new cancers diagnosed in Scotland in $2008 .{ }^{8}$ This may reflect a higher public awareness of melanoma combined with a lack of diagnostic tests available within primary care to help diagnose or exclude melanoma.

The age distribution of patients referred with suspected cancer was more widely spread than expected. A greater proportion of younger patients were referred urgently with suspected cancer when compared with nationally published data on age at first diagnosis (Figure 4). It is therefore not surprising that younger patients referred with suspected cancer were much less likely to have a cancer diagnosed than older patients (Figure 5).

A high proportion of referrals (90.9\%) were self-reported as complying with the national cancer referral guidelines. Of those referrals considered to be in accordance with the referral guidelines, $18.1 \%$ had an eventual diagnosis of cancer. However, even in those referrals considered to be outside the national cancer referral guidelines, $7.7 \%$ had a cancer diagnosed (Figure 6). It therefore appears that there are other important factors, not captured within the national referral guidelines, which have prompted GPs to refer these patients urgently.

\section{Strengths and limitations}

By engaging with 562 different GP practices across nine health boards, there is a risk of variable data capture, depending on the thoroughness with which individual GPs reviewed their clinical notes and recorded the patient data. The development of clear guidance for data collection, a standardised 'locked' electronic reporting template, and the rigorous checking of all data submitted will have helped to reduce this variability. Despite the methods employed to maximise data quality and consistency, there were still some aspects of the data that made analysis and inference difficult. Patient age was a mandatory field, yet for 65 patients $(0.3 \%)$ no age was provided. Data on these 65 patients were therefore excluded from analysis relating to age.

Although records were excluded where the suspected cancer field was left blank, there were still cases where the cancer suspected was entered as either not certain', 'other', or 'no information' (that is, it was not possible to discern from the case notes). Where the cancer suspected is not a specified cancer, interpretation of corresponding information (such as referral within guidelines) is difficult. Therefore, cases where the diagnosis was 'other', 'not certain', or 'no information' were discounted when analysing compliance with guidelines, as their inclusion may skew results.

\section{Comparison with existing literature}

Differences in referral rates between GP practices have been demonstrated for colorectal cancer ${ }^{9}$ however, the authors are unaware of any published research examining and quantifying GP referral patterns and outcomes for all suspected cancers within a UK setting.

Age-related bias in the management of cancer has been previously highlighted in the treatment of breast cancer, with women over the age of 65 years being less likely to receive standardised care than younger women. ${ }^{10}$

Despite the widespread use of referral guidelines by GPs in Scotland, there has only been limited research into the evidence base for compliance with such guidelines. Previous research has examined the diagnostic yield of cancer referrals within an acute hospital setting, and has demonstrated a low predictive power of referral guidelines for colorectal, lung, ovarian, and prostate cancer. ${ }^{11}$ It is thought that referral guidelines may fast-track 
patients with more advanced cancers, possibly to the detriment of other patients whose cancer may be more amenable to curative treatment. ${ }^{12}$

The evidence base of cancer-referral guidelines for both colorectal and lung cancer has been shown to be poor, with some referral recommendations ffor example; abdominal pain for colorectal cancer and unexplained dyspnoea for lung cancerl that do not have any primary care evidence to back them up. 13,14

There is, however, an increasing body of evidence on the positive predictive value of some symptoms, signs, and non-diagnostic tests, for example, rectal bleeding, irondeficiency anaemia, haematuria, evidence of malignancy on rectal examination, haemoptysis, dysphagia, and postmenopausal bleeding. ${ }^{15}$

\section{Implications for research and practice}

The wide variation in referral rate between GPs in Scotland warrants further evaluation. There is no accepted normal referral rate' for suspected cancer and more work is needed in this area.

These results suggest that older people are less likely to be referred urgently with suspected cancer when compared with the younger age groups, despite cancer being more common in older people. Cancerrelated guidelines and education need to reflect this.

A considerable number of referrals (7.7\%) deemed to be outside the national guidelines resulted in a diagnosis of cancer, suggesting that there were other presenting features particular to these patients that alerted the GP to the possibility of cancer. Further research into the likelihood of specific symptoms representing cancer has the potential to inform and improve the usefulness of cancer-referral guidelines in the future. ${ }^{15,16}$ However, it is also important to recognise the limitations with any set of clinical guidelines. Good clinical acumen may allow GPs to identify subtle symptom patterns or early signs of cancer before the more classic 'red flag' symptoms that may indicate more advanced cancer.

Finally, further work is required to examine the cost benefit of wider GP access to currently restricted investigations such as endoscopy, CT, and MRI. 


\section{REFERENCES}

1. Berrino F, De Angelis R, Sant M, et al; EUROCARE Working group. Survival for eight major cancers and all cancers combined for European adults diagnosed in 1995-1999: results of the Eurocare 4 study. Lancet Oncol 2007; 8(9): $773-783$

2. Richards MA. The National Awareness and Early Diagnosis Initiative in England: assembling the evidence. Br J Cancer 2009; 101(Suppl 2): S1-S4.

3. Macleod U, Mitchell ED, Burgess C, et al. Risk factors for delayed presentation and referral of symptomatic cancer: evidence for common cancers. Br J Cancer 2009; 101(Suppl 2): S92-S101.

4. Scottish Executive Health Department. Scottish referral guidelines for suspected cancer. Edinburgh: Scottish Executive Health Department, 2007.

5. NICE. Referral guidelines for suspected cancer. London: National Institute for Health and Clinical Excellence, 2005.

6. Scottish Government. Scottish referral guidelines for suspected cancer. Quick reference guide. Edinburgh: Scottish Government, 2009.

7. Baughan $\mathrm{P}, \mathrm{O}^{\prime}$ Neill B, Fletcher E. Auditing the diagnosis of cancer in primary care: the experience in Scotland. Br J Cancer 2009; 101(Suppl 2): S87-S91.

8. Information Services Division. NHS National Services Scotland. Cancer Incidence in Scotland (2008). Edinburgh: Information Services Division, 2010.
9. John $\mathrm{SK}$, Jones OM, Horseman N, et al. Inter general practice variability in use of referral guidelines for colorectal cancer. Colorectal Dis 2007; 9(8): $731-$ 735.

10. K Lavelle, C Todd, A Moran, et al. Non-standard management of breast cancer increases with age in the UK: a population based cohort of women $>65$ years. Br J Cancer 2007; 96(8): 1197-1203.

11. Allgar V, Neal RD, Ali N, et al. Urgent general practitioner referral for suspected lung, colorectal, prostate and ovarian cancer. Br J Gen Pract 2006; 56(526): 355-362

12. Neal RD, Allgar VL, Ali N, et al. Stage, survival and delay in lung, colorectal, prostate and ovarian cancer: comparison between diagnostic routes. $\mathrm{Br} J \mathrm{Gen}$ Pract 2007; 57(536): 212-219.

13. Hamilton W, Sharp D. Diagnosis of colorectal cancer in primary care: the evidence base for guidelines. Fam Pract 2004; 21(1): 99-106.

14. Hamilton W, Sharp D. Diagnosis of lung cancer in primary care: a structured review. Fam Pract 2004; 21(6): 605-611.

15. Shapley M, Mansell G, Jordan JL, Jordan KP. Positive predictive values of $\leq 5 \%$ in primary care for cancer: systematic review. Br J Gen Pract 2010; 60(578): 681-688.

16. Hamilton W. The CAPER studies: five case-control studies aimed at identifying and quantifying the risk of cancer in symptomatic primary care patients. $\mathrm{Br} J$ Cancer 2009: 101(Suppl 2): S80-S86. 